\title{
Evaluation de clones de colatiers (Cola nitida [Vent.] Schott et Endlicher) pour leur tolérance à la maladie du balai de sorcière du colatier en Côte d'Ivoire
}

\author{
Nadré A. GBEDIE ${ }^{1,3^{*}}$, Kouakou T. KOUADIO ${ }^{1}$, Yaya OUATTARA ${ }^{3,4}$, \\ Klotioloma COULIBALY ${ }^{2}$, Bouadou BONSSON ${ }^{3}$, Drolet J.M. SERY ${ }^{3}$, \\ Hyacinthe N. LEGNATE ${ }^{3}$ and Jules Z. KÉLI ${ }^{3}$
}

\author{
${ }^{1}$ Institut National Polytechnique Félix Houphouët-Boigny (INP-HB) de Yamoussoukro, Département de \\ Formation et de Recherche Agriculture et Ressources Animales, Laboratoire de Phytopathologie et de Biologie \\ Végétale, Unité Mixte de Recherche et d'Innovation en Sciences Agronomiques et Génie Rural, \\ B.P. 1093 Yamoussoukro/Côte d'Tvoire. \\ ${ }^{2}$ Centre National de Recherche Agronomique (CNRA), Station de Recherche de Divo, \\ B.P. 808 Divo/Côte d'Tvoire. \\ ${ }^{3}$ Centre National de Recherche Agronomique (CNRA), Station de Recherche de Man, \\ B.P. 440 Man/Côte d'Ivoire. \\ ${ }^{4}$ Université Nangui Abrogoua, Laboratoire de Biologie et Amélioration des Productions végétales, \\ 02 BP 801 Abidjan 02, Côte d'Ivoire. \\ *Auteur correspondant ; E-mail : ngbedie07@gmail.com; Tel : (+225) 08354313
}

\section{RESUME}

La maladie du balai de sorcière du colatier est la maladie la plus importante du colatier car elle engendre d'énormes pertes de production. L'objectif principal de cette étude était d'identifier des clones de colatiers d'origine ivoirienne qui affichent le meilleur profil de tolérance à la maladie du balai de sorcière et ayant des paramètres de rendement intéressants. Pour atteindre cet objectif, un dispositif expérimental en bloc complètement randomisé à 3 répétitions a été utilisé et le facteur étudié est le clone avec 13 modalités. Les résultats obtenus montrent que 5 clones (clones 305, 314, 318, A2 et A3) n'ont pas subi d'attaque de la maladie. Les clones 311, 313, 315, 321 et 322 ont été moyennement attaqués. Sur la base des paramètres de rendement tels que le poids des noix par follicule et le nombre de noix par follicule, les clones 313 et 323 ont produit les plus grosses noix dont le poids moyen varie entre $31,98 \pm 11,60 \mathrm{~g}$ et $34,97 \pm 8,54 \mathrm{~g}$ et le clone 316 a donné un plus grand nombre de noix par follicule (en moyenne, 10,63 $\pm 1,55$ noix par follicules). Une classification combinant le niveau de tolérance des clones et les paramètres de rendement étudiés ont permis d'identifier les clones 313,322 et 323 comme étant les meilleurs.

(C) 2019 International Formulae Group. All rights reserved.

Mots clés : Maladie du balai de sorcière, colatier, pertes de production, rendement. 


\title{
Assessment of kola clones (Cola nitida [Vent.] Schott and Endlicher) for tolerance to witch's broom disease in Côte d'Ivoire
}

\begin{abstract}
Witch's broom disease is the most important disease of kola tree because it causes enormous production losses. The main objective of this study was to identify kola clones of Ivorian origins that have the best tolerance profile for witch's broom disease and have interesting yields parameters. The experimental setup used is a completely randomized block with 3 repetitions and the studied factor is genotype with 13 modalities. The results obtained show that 5 clones (clones 305, 314, 318, A2 and A3) have not been attacked. Clones 311, 313, 315, 321 and 322 were moderately attacked. Clones 322 and 323 produced large follicles with average weights of $501.73 \pm 153.75 \mathrm{~g}$ and $508.23 \pm 131.41 \mathrm{~g}$ respectively. Based on yield parameters such as nuts weight per follicle and number of nuts per follicle, clones 313 and 323 produced the largest nuts with average weights ranging from $31.98 \pm 11.60 \mathrm{~g}$ to $34.97 \pm 8.54 \mathrm{~g}$ and clone 316 produced a greater number of nuts per follicle (on average, $10.63 \pm 1.55$ nuts per follicle). A classification combining the tolerance level of the clones and yield parameters studied identified clones 313,322 and 323 as the best.
\end{abstract}

(c) 2019 International Formulae Group. All rights reserved.

Keywords: Witch's broom disease, kola tree, crop loss, yield.

\section{INTRODUCTION}

Le colatier est un arbre de la forêt tropicale africaine qui appartient à la famille des Malvacées (Whitlock et al., 2001) et au genre Cola (Bodard, 1962). Ce genre comprend plus de 140 espèces (Adenuga et al., 2012) et l'espèce cultivée en Côte d'Ivoire est Cola nitida.

La production de noix de cola en Côte d'Ivoire est estimée à plus de 100000 t/an (Aloko-n'guessan, 2000). Ce niveau de production est inférieur à la demande, aussi bien pour le commerce local que pour le commerce international (Oluwalana et al. 2016). Parmi les facteurs qui limitent cette production, figurent l'absence de matériel végétal performant (Mbete et al., 2011), les difficultés des producteurs à accéder à une assistance technique (Berté, 2009), les fréquentes attaques du colatier et de la noix de cola par des insectes tels que Balanogastris colae et Paremydica sp. et par la maladie du balai de sorcière qui font peser des menaces sur la durabilité de l'exploitation de cette spéculation (Aloko-n'guessan, 2000).

La maladie du balai de sorcière est la maladie la plus importante du colatier car elle peut causer des pertes de production allant jusqu'à $60 \%$ selon la sévérité. Ce genre de maladie affecte les plantes de la famille des Malvaceae (dont le cacaoyer et le cupuaçu), des Bixaceae (Bixa orellana), des Bignoniaceae (les lianes), des Solanaceae et des Malpighiaceae (Resende et al., 2000). Les symptômes observés sur ces plantes s'apparentent à ceux observés sur le colatier. Les tissus méristématiques de différents organes du colatier dont les bourgeons floraux sont infectés. L'infection des fleurs est suivie du passage de la structure florale à la formation de fleurs anormales (taille, nombre de pièces florales, nombre d'ovaires et de stigmates) (Figure 1). La croissance hypertrophique des méristèmes végétatifs infectés, qui aboutit à la formation d'une structure en forme de balai (d'où le nom de la maladie) constitue le symptôme le plus caractéristique de la maladie (Micheli, 2009) (Figure 2). L'hypertrophie du tissu végétal et la prolifération de pousses axillaires et des tiges anormales constituent le stade de «balais verts » et la phase nécrotrophe qui correspond à la nécrose et à la mort de tissus infectés constitue le stade de «balais secs » (Almeida et al., 2017). A ce jour, l'agent causal de cette maladie sur le colatier reste encore inconnu. 
Afin d'améliorer le matériel végétal, des études antérieures réalisées en Côte d'Ivoire avaient permis de constituer des collections (Ouattara et al., 2018). Ces collections mises en place par le programme d'amélioration de la productivité de la noix de cola de l'Institut de Recherche du Café et Cacao et autres plantes stimulantes (IRCC), dans les années 1970-1980, étaient constituées de plusieurs clones d'origine nigériane, guinéenne, sénégalaise et ivoirienne (Bonsson, 1983). Ce matériel végétal n'a, jusqu'à la présente étude, fait l'objet d'une évaluation vis-à-vis de l'une des maladies dévastatrices du colatier qui est celle du balai de sorcière.

La présente étude est proposée pour combler ce déficit d'information. Son objectif principal est d'identifier des clones de colatier d'origine ivoirienne qui affichent le meilleur profil de tolérance face à la maladie du balai de sorcière et ayant des paramètres de rendement intéressants.

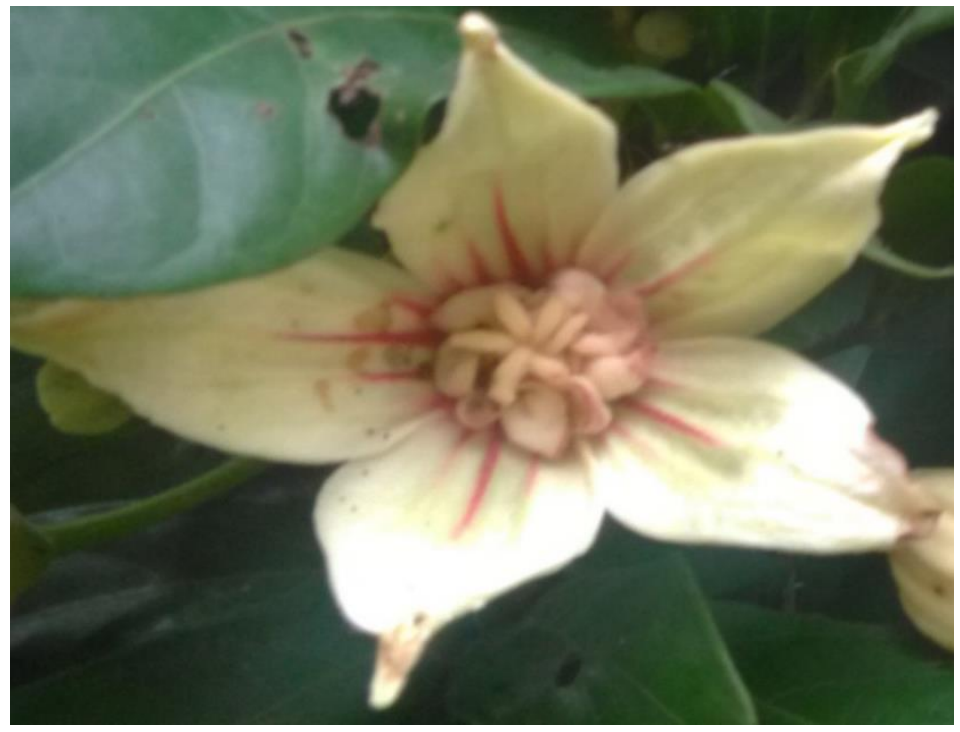

Figure 1 : Fleur anormale due à la maladie du balai de sorcière.
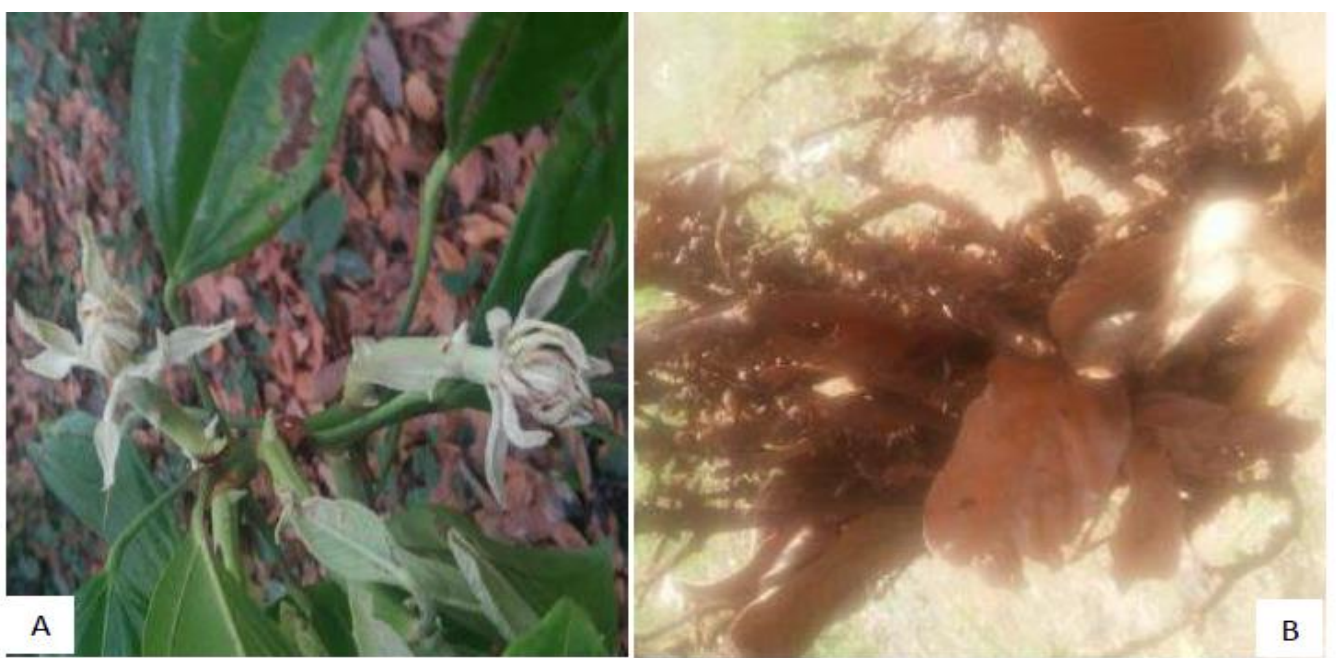

Figure 2 : Symptômes de la maladie du balai de sorcière du colatier au stade de «balai vert » (A) et au stade de « balai sec » (B). 


\section{MATERIEL ET METHODES \\ Site de l'étude}

L'étude a été menée en 2015 et 2016, à la station de recherche du CNRA de Divo $\left(5^{\circ} 50^{\prime} 27.8^{\prime \prime} \mathrm{N}, \quad 5^{\circ} 21^{\prime} 30.1^{\prime \prime} \mathrm{W}\right)$ durant la période de fructification des arbres. Cette station, située à $17 \mathrm{~km}$ de la ville de Divo, se caractérise par quatre saisons dont une grande saison de pluie de Mars à Juin, pendant laquelle tombe les $2 / 3$ des précipitations annuelles, une petite saison de pluie de Septembre à Novembre, une grande saison sèche de Décembre à Février, et une petite saison sèche de Juillet à Août. La pluviométrie moyenne annuelle est de 1354 $\mathrm{mm}$. Les températures varient de 25 à $30^{\circ} \mathrm{C}$ (Ouattara et al., 2018).

\section{Matériel végétal}

Le matériel végétal testé était constitué de 13 clones de colatiers d'origine ivoirienne. Ces clones ont été plantés en 1964 à la station de recherche du Centre National de Recherche Agronomique (CNRA) de Divo sur la parcelle D3/1. Il s'agit des clones 305, $306,311,313,314,315,316,318,321,322$, 323, A2 et A3.

\section{Dispositif expérimental}

Le dispositif expérimental utilisé sur le site est un bloc en randomisation totale. Le facteur étudié est le clone avec 13 modalités. Les clones de colatier ont été plantés aux écartements de $8 \mathrm{~m}$ entre les lignes et de $4 \mathrm{~m}$ sur la ligne, soit à la densité de 312 pieds/ha.
Les blocs étaient disposés de façon perpendiculaire à la pente. $\mathrm{La}$ parcelle élémentaire était constituée d'un arbre par clone de colatier répété trois (03) fois dans la parcelle. Au total, 39 pieds de colatier ont été utilisés pour cette expérimentation.

L'évaluation de la sévérité de la maladie a été faite à la période de fructification de chaque année et pour les mesures agronomiques, 30 follicules arrivés à maturité ont été observés par clone sur lesdites périodes.

\section{Collecte de données}

La sévérité de la maladie a été notée à l'âge adulte de l'arbre en 2015 et en 2016, pendant la phase de fructification, selon l'échelle présentée dans le tableau 1 . Le poids des noix par follicule (PNF) et le nombre de noix par follicule (NF) ont été également collectés puis mesurés. Une moyenne de chaque paramètre a été déterminée.

\section{Analyse statistique des données}

La normalité des données a été vérifiée par le test de Shapiro-Wilk au seuil de 5\% (Shapiro et Wilk, 1965). L'analyse de variance a été effectuée avec le logiciel SAS 9.4 au seuil de $5 \%$ afin de déterminer si les traitements étaient différents les uns des autres. Une classification hiérarchique ascendante a été réalisée avec le logiciel $\mathrm{R}$ 3.0.3 en prenant en compte tous les paramètres étudiés.

Tableau 1 : Echelle de notation de la sévérité de la maladie du balai de sorcière.

\begin{tabular}{lll}
\hline Note de sévérité & $\begin{array}{l}\text { Description des dégâts sur le colatier } \\
(\% \text { des rameaux attaqués par arbre })\end{array}$ & Niveau de tolérance \\
&
\end{tabular}

\begin{tabular}{lcl}
\hline 0 & Aucun rameau attaqué & Résistant \\
1 & $1-25 \%$ & Très tolérant \\
2 & $26-50 \%$ & Moyennement tolérant/sensible \\
3 & $51-75 \%$ & Sensible \\
4 & $76-100 \%$ & Hautement sensible
\end{tabular}




\section{RESULTATS}

\section{Réaction des clones face à la maladie du balai de sorcière}

L'analyse de variance effectuée sur les notes de sévérité de la maladie du balai de sorcière du colatier (Tableau 2) montre une variation significative des paramètres mesurés suivant le facteur clone. Trois groupes ont été identifiés. Les clones 305, 314, 318, A2 et A3 constituent le groupe n'ayant pas subi d'attaque parasitaire. Le second groupe composé des clones $311,313,315,321$ et 322 ayant une note de sévérité égale à 2 , ont été moyennement attaqués. Les clones les plus attaqués constituent le troisième groupe qui est constitué des clones 306,316 et 323 . Ces derniers ont obtenu des notes de sévérité de 4.

\section{Poids des noix par follicule}

Le poids moyen des noix en fonction de chaque clone testé a été mesuré. L'analyse de variance montre une différence significative entre les clones $(p<0,0001)$. Les clones qui ont produit les plus grosses noix sont les clones 313 et 323 avec des poids moyens de noix respectifs de $31,98 \pm 11,60 \mathrm{~g}$ et $34,97 \pm 8,54 \mathrm{~g}$. Les clones $305,306,311,314$, $315,316,318,321,322$, A2 et $\mathrm{A} 3$ ont des petites noix de poids compris entre $10,63 \pm 1,55 \mathrm{~g}$ et $26,57 \pm 8,24 \mathrm{~g}$ (Tableau 2).

\section{Nombre de noix par follicule}

L'analyse de variance effectuée sur le nombre moyen de noix par follicule a révélé une différence significative $(\mathrm{p}<0,0001)$. Le clone 316 avait en moyenne $10,68 \pm 2,39$ noix par follicule contrairement aux autres clones qui avaient des follicules comportant en moyenne $4,92 \pm 2,69$ à $8,92 \pm 1,61$ noix (Tableau 2).

\section{Classification hiérarchique ascendante des clones en fonction de la sévérité de la maladie et de quelques paramètres de rendement}

La classification hiérarchique ascendante prenant en compte quelques paramètres de rendement étudiés de chaque clone et la sévérité de la maladie du balai de sorcière a permis de mettre en évidence les clones 313,322 et 323 qui possèdent de très bons paramètres de rendement et tolérants à la maladie (Figure 3). Le clone 323 est le clone ayant subi le plus l'attaque parasitaire et présente des paramètres de rendement intéressants, il peut être vulgarisé dans les zones où la maladie est non endémique.

Tableau 2 : Moyenne des caractères fructifères et de la sévérité de la maladie.

\begin{tabular}{lllc}
\hline Clones & NF & PNF & Sévérité \\
\hline 305 & $7,32 \pm 1,81 \mathrm{bcd}$ & $22,56 \pm 6,68 \mathrm{bcd}$ & $0 \mathrm{a}$ \\
306 & $6,28 \pm 2,17 \mathrm{cde}$ & $26,57 \pm 8,24 \mathrm{~b}$ & $4 \mathrm{c}$ \\
311 & $8,08 \pm 2,12 \mathrm{~b}$ & $17,66 \pm 4,02 \mathrm{~d}$ & $2 \mathrm{~b}$ \\
313 & $7,64 \pm 2,67 \mathrm{bc}$ & $31,98 \pm 11,60 \mathrm{a}$ & $2 \mathrm{~b}$ \\
314 & $5,48 \pm 2,48 \mathrm{de}$ & $24,68 \pm 7,52 \mathrm{bc}$ & $0 \mathrm{a}$ \\
315 & $6,24 \pm 2,24 \mathrm{cde}$ & $17,63 \pm 6,30 \mathrm{~d}$ & $2 \mathrm{~b}$ \\
316 & $10,68 \pm 2,39 \mathrm{a}$ & $10,63 \pm 1,55 \mathrm{e}$ & $4 \mathrm{c}$ \\
318 & $5,72 \pm 2,87 \mathrm{de}$ & $18,77 \pm 11,35 \mathrm{~cd}$ & $0 \mathrm{a}$
\end{tabular}




\begin{tabular}{llll}
321 & $4,92 \pm 2,69 \mathrm{e}$ & $21,17 \pm 8,64 \mathrm{bcd}$ & $2 \mathrm{~b}$ \\
322 & $8,90 \pm 2,30 \mathrm{~b}$ & $26,36 \pm 8,45 \mathrm{~b}$ & $2 \mathrm{~b}$ \\
323 & $8,92 \pm 1,61 \mathrm{~b}$ & $34,97 \pm 8,54 \mathrm{a}$ & $4 \mathrm{c}$ \\
$\mathrm{A} 2$ & $5,52 \pm 1,66 \mathrm{de}$ & $22,74 \pm 2,96 \mathrm{bcd}$ & $0 \mathrm{a}$ \\
$\mathrm{A} 3$ & $8,24 \pm 2,20 \mathrm{~b}$ & $20,82 \pm 3,61 \mathrm{bcd}$ & $0 \mathrm{a}$ \\
\hline Moyennes & $7,22 \pm 2,25$ & $22,81 \pm 6,88$ & 1,69 \\
CV (p.c.) & 31,57 & 32,90 & 0,0 \\
probabilité & $<0,0001$ & $<0,0001$ & $<0,0001$
\end{tabular}

$\mathrm{NF}=$ Nombre de noix par follicule $; \mathrm{PNF}=$ Poids des noix par follicule $; \mathrm{CV}=$ Coefficient de variation ; $\mathrm{P}=$ Probabilité $; \mathrm{S}=$ Significatif.

\section{Factor map}

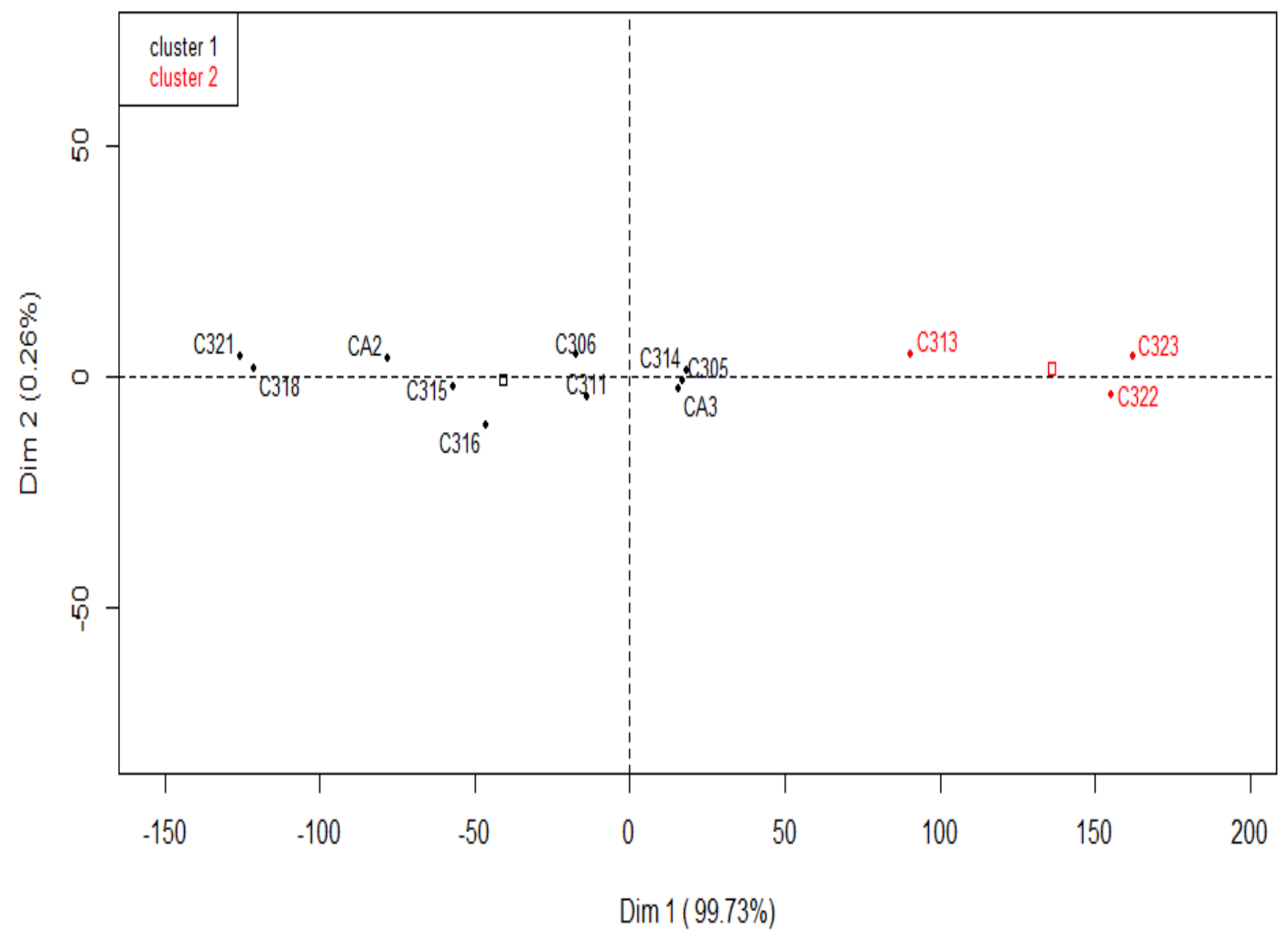

Figure 3 : Classification hiérarchique ascendante des clones en fonction de la sévérité de la maladie du balai de sorcière et de leurs paramètres de rendement. 


\section{DISCUSSION}

L'évaluation de clones de colatiers pour leur tolérance à la maladie du balai de sorcière du colatier a révélé une variabilité de la sévérité de la maladie et des paramètres de rendement suivant le clone.

$\mathrm{Au}$ cours de l'année 2015, les clones $305,314,318, \mathrm{~A} 2$ et $\mathrm{A} 3$ ont été résistants à cette maladie. Les clones 311, 313, 315, 321 et 322 ont été moyennement tolérants et les clones 306, 316 et 323 ont été hautement sensibles à la maladie. L'explication de ces résultats présume de l'influence du génotype sur l'expression de la maladie. Il a été montré par Flor (1971) cité par Bouet et al. (2015) que l'interaction maladie-génotype est régie par la loi « un gène de résistance pour un gène d'avirulence ». L'étude de l'héritabilité constituerait une piste de recherche afin d'élucider cette interaction plante hôtepathogène.

L'attaque de la maladie du balai de sorcière sur les clones testés a été sévère en 2015. Cela pourrait s'expliquer par les conditions climatiques notamment la pluviométrie et l'humidité relative plus favorables en 2015.

La classification hiérarchique ascendante a révélé la diversité des clones testés en fonction de leur tolérance à la maladie du balai de sorcière, du poids des noix par follicule et du nombre de noix par follicule. Les clones 313, 322 moyennement tolérants et le clone 323 , hautement sensible à la maladie du balai de sorcière, présentent des paramètres de rendement intéressants. Ces clones sont tolérants à la maladie du balai de sorcière. Ces résultats sont similaires à ceux obtenus par Bonsson (1983), dans son étude portant sur l'Amélioration de la production de noix de cola en Côte d'Ivoire, qui avait confirmé, sur plusieurs années, le bon comportement relatif des clones 322 et 323 . Ces clones ont produit respectivement en 1981, par exemple, 103 et 111 follicules par arbre contre 32 follicules pour l'ensemble des clones. Cela pourrait justifier leur utilisation dans les programmes d'amélioration génétique et de sélection car leur tolérance pourrait être améliorée.
L'évaluation des paramètres de rendement des 13 clones de colatiers a mis en évidence l'existence d'une importante variabilité. Cette variabilité a été ensuite structurée en deux (02) grands groupes distincts caractérisés par les clones qui possèdent les paramètres de rendement intéressants et ceux qui ne les possèdent pas. Cette variabilité serait due à la variabilité intra-génétique du matériel végétal testé.

Les résultats de cette étude ont clairement mis en évidence la variabilité de la sévérité de la maladie du balai de sorcière sur les clones de colatiers testés à la station de recherche du CNRA de Divo. De plus, les génotypes possédant des caractères fructifères souhaitables et présentant une forme de tolérance stable peuvent être utilisés dans les programmes d'amélioration génétique et de sélection du colatier.

\section{Conclusion}

L'étude menée à la station de recherche du CNRA de Divo a permis d'identifier des clones dotés de très bonnes aptitudes à résister à la maladie du balai de sorcière. Il s'agit des clones 305, 314, 318, A2 et A3. En combinant le niveau de résistance de ces clones face à la maladie du balai de sorcière et leurs paramètres de rendement, les clones 313, 322 et 323 se révèlent les meilleurs. Cette étude doit être élargie à d'autres génotypes afin d'identifier ceux qui sont résistants. La mise en place de tests multi-locaux permettra de voir l'interaction génotype-environnement et au mieux, de ressortir (si possible) une existence d'une diversité d'isolats ou race du pathogène.

\section{CONFLIT D'INTERETS}

Les auteurs déclarent qu'ils n'ont aucun conflit d'intérêts.

\section{CONTRIBUTIONS DES AUTEURS}

YA a mis en place et assuré le suivi de l'essai. Il a également contribué à la collecte des données. NAG a élaboré le manuscrit. KTK, BB, DJMS, CK, LNH et KZJ ont apporté leur contribution à travers la correction du manuscrit. 


\section{REMERCIEMENTS}

Nous adressons nos sincères remerciements au Fonds Interprofessionnel pour la Recherche et le Conseil Agricoles (FIRCA) pour le financement de cette étude.

\section{REFERENCES}

Adenuga OO, Mapayi EF, Olasupo FO, Olaniyi OO, Oyedokun AV. 2012. Nigeria's Cola genetic ressources: the need for renewed exploration. Asian $J$. Agri. Sci., 4: 177-182.

Aloko-N'guessan J. 2000. Cola, espace et sociétés : Étude de la géographie sociale et culturelle de la filière cola au marché de Gros de Bouaké (Côte d'Ivoire). Rev. CAMES - Série B, (2) : 25 - 35.

Berté. 2009. Etat des lieux de la filière cola en Côte d'Ivoire. Rap. FIRCA, p. 87.

Almeida DSM, Gramacho KP, Cardoso THS, Micheli F, Alvim FC, Pirovani CP. 2017. Cacao Phylloplane: The First Battlefield against Moniliophthora perniciosa, Which Causes Witches' Broom Disease. Phytopathology, 107: 864-871. DOI: http://dx.doi.org/10.1094/PHYTO-0616-0226-R

Bodard M. 1962. Contribution à l'étude systématique du genre Cola nitida en Afrique Occidentale. Ann. Fac. Sci., 7:17 $-24$.

Bonsson B. 1983. Amélioration de la production de la noix de cola en Côte d'Ivoire: Principe de sélection. Café Cacao Thé, 27(4) : 283 - 297.

Bouet A, Gbédié NA, Boka A, Kouassi N. 2015. Evaluation des variétés de riz prometteuses pour la résistance à quelques contraintes biotiques majeures et pour leurs performances agronomiques en Côte d'Ivoire. Int. J. Biol. Chem. Sci., 9(4): 2041-2056. DOI: http://dx.doi.org/10.4314/ijbcs.v9i4.27

Flor HH. 1971. Current statut of gene for gene concept. Annual Review of Phytopathology, 9: 275-296.
Mbete P, Makosso S, Lelou B, Douh C, Ngokaka C. 2011. Essai de multiplication végétative du Colatier (Cola nitida) via la technique du marcottage au Congo Brazzaville. Journal of Applied Biosciences, 37: 2485 $-2490$.

Micheli F. 2009. Study of the Theobroma cacao-Moniliophthora perniciosa interaction. Biochimie [q-bio.BM]. Thèse de doctorat, Université Paris Sud Paris XI, p.86.

Oluwalana EOA, Okojie LA, Ashaolu FO, Olaniran VO. 2016. An analysis of yields of kolanuts production and marketing in Shagamu, Ogun State, Nigeria. Journal of Humanities, Social Science and Arts, 11(1): 88-103.

Ouattara Y, Bonsson B, Traoré MS, Gbédié NA, Akaffou DS, Sié RS, Keli ZJ. 2018. Évaluation de la diversité agromorphologique d'accessions de colatiers (Cola nitida (Vent.) Schott et Endlicher) collectées au Sud et au Sud-Ouest de la Côte d'Ivoire. Journal of Applied Biosciences, 122: 12296-12308. DOI: https://dx.doi.org/10.4314/jab.v121i1.10

Resende ML, Gutemberg BA, Silva LH, Niella GR, Carvalho GA, Santiago DV, Bezerra, JL. 2000. Crinipellis perniciosa proveniente de um novo hospedeiro Heteropterys acutifolia é patogênico ao cacaueiro. Fitopatol. Brasil., 25(10): 88 91.

Shapiro SS, Wilk MB. 1965. An analysis of variance test for normality (complete samples). Biometrika, 52: 591 - 611.

Whitlock BA, Bayer C, Baum DA. 2001. Phylogenetic relationships and floral of the Byttnerioideae (Sterculiaceae or Malvaceae S.1.) based on sequences of the chloroplast gene, ndhF. Systematic Botany, 26 (2): 420-437. 